\title{
Estratégias de atuação do nutricionista em consultoria alimentar e nutricional da família
}

\author{
Dietitian's procedures in food and \\ nutrition consultancy for the family
}

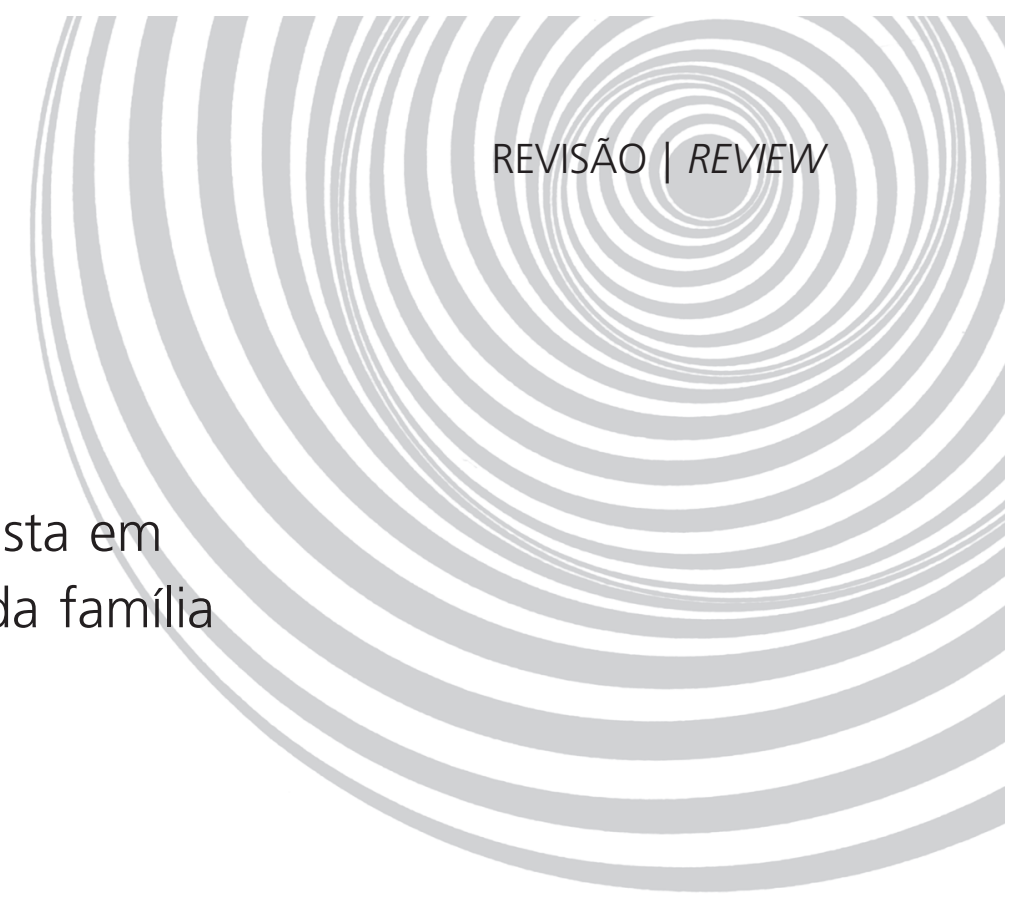

Patrícia Afonso de ALMEIDA-BITTENCOURT ${ }^{1}$

Paula Severino Azambuja RIBEIRO'

Maria Margareth Veloso NAVES 2

\section{RES U M O}

O objetivo deste trabalho é apresentar princípios e estratégias de atuação do nutricionista em consultoria alimentar e nutricional da família. O especialista nesta área, denominado personal dieter, tem por objetivo principal a educação nutricional da família. A atuação do personal dieter é baseada em três princípios fundamentais: envolvimento de todos os membros da família, promoção da alimentação saudável e aperfeiçoamento das técnicas dietéticas usadas no preparo dos alimentos. Para isso, o nutricionista realiza visitas domiciliares para conhecer a realidade da família e propõe mudanças dos hábitos alimentares por etapas. 0 protocolo de intervenção adaptado a cada realidade inclui, entre outras atividades, elaboração de cardápio e de lista de compra de alimentos, prescrição de dieta individualizada e treinamento de cozinheira. O conhecimento detalhado dos comportamentos, hábitos e práticas relacionados à alimentação da família favorece uma intervenção personalizada. Isto é essencial para a adoção de hábitos alimentares saudáveis e garante maior eficácia do profissional nutricionista no cuidado à nutrição e à saúde da família.

Termos de indexação: Dietética. Educação alimentar e nutricional. Hábitos alimentares. Nutricionista. Saúde da família.

\section{A B S T R A C T}

The objective of this work is to present the principles and strategies of a dietitian in food and nutrition consultancy for the family. Also known as a personal diet specialist, these dietitians focus mainly on the nutrition education of the family. Their practice is based on three fundamental principles: participation of the whole family, promotion of healthy eating habits and optimization of dietary techniques used in the food preparation. To achieve these goals, the dietitian visits families in their households in order to learn about the family's lifestyle and suggests changes in their food habits one at a time. The intervention strategy is personalized

1 VP Consultoria Nutricional e Educação. São Paulo, SP, Brasil.

2 Universidade Federal de Goiás, Faculdade de Nutrição. R. 227, Quadra 68, Setor Leste Universitário, 74605-080, Goiânia, GO, Brasil. Correspondência para/Correspondence to: M.M.V. NAVES.E-mail: <mnaves@fanut.ufg.br>. 
920 | P.A. ALMEIDA-BITTENCOURT et al.

and includes, among other things, designs the menu and drafts a groceries shopping list, prescribes an individualized diet and trains the cook. Detailed knowledge of the family's food habits, behaviors and lifestyle promotes a personalized intervention. This is vital for the acquisition of healthy eating habits and guarantees greater dietitian's effectiveness in the nutrition and health care of the family.

Indexing terms: Dietetics. Food and nutrition education. Food habits. Nutritionist. Family health.

\section{N T R O D U ÇÃ O}

O ano de 2007 constituiu um marco na história da nutrição no Brasil, pois foi o ano em que se completou 40 anos de regulamentação da profissão de nutricionista. Atualmente, o País está com quase 50 mil profissionais atuando em diversas áreas. A área de maior concentração é a Nutrição Clínica $(41,7 \%)$, seguida da área de Alimentação Coletiva, na qual atuam $32,2 \%$ dos nutricionistas ${ }^{1}$.

Vasconcelos ${ }^{2}$ descreve a profissão do nutricionista no Brasil em quatro fases. A primeira fase, denominada pelo autor de "Emergência da profissão", compreende o período entre 1939 e 1949, em que ocorreu a abertura do primeiro curso de nutrição no País. A segunda fase, da "Consolidação da profissão", que considera os anos de 1950 a 1975, foi caracterizada pela luta em prol da regulamentação da profissão. A terceira fase refere-se à "Evolução da profissão" (1976 a 1984), em que ocorreu a criação dos Conselhos Federal e Regionais de Nutricionistas e de vários cursos de graduação em Nutrição. A quarta fase, da "Reprodução ampliada" (1985 a 2000), caracterizou-se por importantes eventos técnico-científicos e pelo crescente processo de mobilização e politização da categoria. Nesta última fase, destaca-se a ampliação dos campos de atuação profissional, o que tem gerado uma demanda pela educação continuada do nutricionista, visando à aquisição de novos conhecimentos e ao domínio de novas ferramentas tecnológicas. Em contrapartida, a expectativa da população em relação à nutrição e à atuação do nutricionista tem aumentado, uma vez que a nutrição tem se tornado um dos temas mais especulativos relacionados às Ciências da Saúde.
Por outro lado, o padrão alimentar nas áreas metropolitanas do Brasil tem se caracterizado pelo consumo excessivo de produtos alimentícios industrializados, em detrimento da ingestão de alimentos regionais e tradicionais, como a farinha de mandioca, o arroz e o feijão, e por um consumo reduzido de frutas e hortaliças ${ }^{3,4}$. As mudanças no padrão alimentar da população brasileira, o aumento da incidência de doenças e agravos não transmissíveis e a influência de fatores ambientais e da mídia constituem fatores que têm contribuído para que as pessoas busquem alternativas de melhoria da qualidade de vida. Dentre elas, destaca-se a adoção de uma alimentação saudável, cuja relação com a saúde tem sido reconhecida por consumidores de diferentes seguimentos da sociedade ${ }^{4,5}$.

Os consultórios particulares de Nutrição, em geral, atendem à demanda de pacientes que não estão hospitalizados, mas que necessitam de uma intervenção nutricional. A prática da educação nutricional tem mostrado que apenas orientar acerca de uma alimentação balanceada e equilibrada não garante a mudança de hábitos alimentares e o alcance de resultados mais duradouros. São muitos os fatores, de ordem psicológica e sociocultural, que dificultam a adesão à dieta, como, por exemplo, a enorme oferta de alimentos industrializados, a pouca disponibilidade de alimentos saudáveis em casa, a falta de envolvimento e orientação dos familiares, a falta de motivação e de tempo, a vida social ativa, entre outros ${ }^{4,6,7}$.

Nesse contexto, o atendimento nutricional diferenciado surge como uma necessidade de adaptação do nutricionista aos padrões de exigência da sociedade atual, para garantir maior eficácia em sua prática profissional. Este novo campo de atuação, em que o nutricionista é deno- 
minado Personal dieter, compreende uma consultoria domiciliar que oferece um plano alimentar completo, envolvendo todas as etapas do processo alimentar, desde a aquisição do alimento até seu consumo, e que contemplam um programa de mudanças gradativas na rotina da família, no sentido da adoção de uma alimentação saudável ${ }^{8}$. Sendo assim, este profissional se caracteriza pelo atendimento a famílias que realizam as refeições principais (desjejum, almoço e jantar) no domicílio. O envolvimento da família no processo de mudança de hábitos alimentares constitui uma estratégia que pode contribuir significativamente para a adesão dos clientes a esse processo e o alcance dos resultados esperados, de forma definitiva ${ }^{6,8}$.

Este trabalho tem o objetivo de apresentar princípios e estratégias de atuação do nutricionista, como personal dieter, em consultoria alimentar e nutricional da família.

\section{Princípios}

O comportamento alimentar está fortemente ligado ao estilo e aos hábitos de vida dos indivíduos ${ }^{7}$. O estilo de vida típico ocidental, por sua vez, conduz a hábitos inadequados, que constituem fatores de risco de doenças e agravos não transmissíveis, como obesidade, hipertensão arterial, câncer e diabetes mellitus ${ }^{4}$. Além disso, a dieta inadequada pode causar doenças carenciais, sobretudo de micronutrientes, independentemente das condições socioeconômicas dos indivíduos ${ }^{9}$. Em contrapartida, relatos de literatura indicam que a adesão de pacientes a mudanças no estilo de vida visando à prevenção e ao tratamento dessas doenças, como a adesão a uma dieta que envolve restrições alimentares, é difícil de ser conseguida e, em geral, é baixa ${ }^{10,11}$.

A conduta nutricional considerada muito complicada, como, por exemplo, quando a dieta proposta não se adapta à rotina do cliente ou da família, a dificuldade de acesso a alimentos apropriados ou esforços extras requeridos no preparo do alimento são fatores suficientes para justificar a não-adesão ao tratamento. Por outro lado, a prescrição do plano alimentar de acordo com a realidade do cliente constitui estratégia determinante do sucesso da intervenção ${ }^{11-13}$. A satisfação diante da informação transmitida, as características da conduta nutricional e a postura do profissional na residência do cliente são fatores que também influenciam a adesão às mudanças propostas.

Dessa forma, a assistência domiciliar personalizada requer uma reestruturação do protocolo tradicional de atendimento nutricional, uma vez que a atuação do personal dieter é baseada em três princípios fundamentais: envolvimento de todos os membros da família, promoção da alimentação saudável e aperfeiçoamento das técnicas dietéticas usadas no preparo dos alimentos. Além disso, outro componente que norteia a atuação do profissional refere-se ao resgate do hábito de se alimentar em família, um aspecto considerado importante na adoção de hábitos alimentares saudáveis e na promoção da saúde e da qualidade de vida ${ }^{7,14}$.

\section{Estratégias}

\section{Aspectos gerais}

Algumas estratégias são características do atendimento nutricional personalizado, indispensáveis para que a família mude seus hábitos alimentares: reunir os membros da família envolvidos no processo, estabelecer metas de acordo com as prioridades da família, introduzir cardápios com novos alimentos, conforme o estilo de vida da família, utilizar receitas práticas e funcionais e orientar quanto à lista e à aquisição de alimentos.

A clientela inicial de um personal dieter pode ser captada por meio de pequenas reuniões em parcerias com lojas, prédios, condomínios ou com qualquer outro grupo de pessoas. Nas reuniões devem ser ministradas palestras sobre nutrição e qualidade de vida e, em seguida, são divulgados os serviços oferecidos pelo profissional. Paralelamente a esta ação, deve ser construído um banco de dados de cardápios e receitas. Para 
a avaliação e o planejamento dietético, é fundamental o uso de software de Nutrição, para auxiliar na montagem de cardápios, no cadastro de receitas e de insumos e no planejamento de dietas individuais. As receitas devem ser testadas pelo nutricionista e aprovadas pelos clientes, e os cardápios podem ser elaborados conforme as estações do ano ou segundo suas características específicas, como cardápios com ação detoxificante, vegetariano, por grupos de alimentos, para diabéticos etc. ${ }^{8}$ Alguns aspectos importantes valem ser reforçados na elaboração dos cardápios: variação e combinação dos ingredientes e pratos, disponibilidade dos alimentos (safra e mercado), praticidade das receitas (técnicas de preparo e mão-de-obra disponível), custo acessível e valor nutricional adequado e balanceado, inclusive em micronutrientes 8,15 .

Outro serviço que facilita a execução do cardápio e a adesão às intervenções propostas é o treinamento da cozinheira. Este treinamento tem o objetivo de orientar quanto às técnicas de pre- paro dos alimentos que fazem parte do cardápio. É importante que o nutricionista tenha um bom conhecimento de técnicas dietéticas, incluindo técnicas que garantam um melhor aproveitamento dos nutrientes, para que possa orientar com segurança e eficácia.

Embora o serviço do personal dieter esteja vinculado ao atendimento da família, pode haver a necessidade de prescrição de dieta individualizada e de elaboração de esquemas alimentares e orientações específicas para membros que realizam alguma refeição principal fora do domicílio, ou para situações especiais, como viagens.

\section{Protocolo de atendimento nutricional proposto}

A partir das informações coletadas por meio de um inquérito familiar detalhado (Quadro 1), são estabelecidas as mudanças necessárias dos hábitos alimentares visando à promoção da saúde, com metas definidas conforme o objetivo principal

Quadro 1.Tipos de informações a serem coletadas pelo personal dieter mediante inquérito familiar.

\begin{tabular}{|c|c|}
\hline Tipo de Informação & Especificação \\
\hline Identificação & Nome de todos os moradores da casa, idade, escolaridade e contatos \\
\hline Serviços oferecidos & $\begin{array}{l}\text { Cardápio; treinamento de cozinheira; lista de supermercado; prescri- } \\
\text { ção de dieta individualizada; aulas de educação nutricional }\end{array}$ \\
\hline Período de acompanhamento & Dois meses ou mais \\
\hline Freqüência de visitas & Semanal, quinzenal ou mensal \\
\hline Objetivo principal da família & Perda de peso, variação de cardápio, qualidade de vida etc. \\
\hline Prática de atividade física & Quem pratica atividade física, tipo de atividade e freqüência \\
\hline Práticas alimentares da família & $\begin{array}{l}\text { Refeições realizadas em conjunto, aversões e restrições, o que vai à } \\
\text { mesa, líquido às refeições etc. }\end{array}$ \\
\hline Informações relacionadas à cozinheira & $\begin{array}{l}\text { Nome, especialidade, técnicas dietéticas usadas, cardápios, técnicas } \\
\text { de higiene empregadas (uso de touca, luva, uniforme), organização } \\
\text { e desempenho na cozinha, tempero (salgado, insípido, oleoso) etc. }\end{array}$ \\
\hline $\begin{array}{l}\text { Afecções e terapias relacionadas com o estado } \\
\text { nutricional da família }\end{array}$ & Alergias, estado imunológico, medicamentos e suplementações \\
\hline Compra de alimentos & $\begin{array}{l}\text { Supermercado, açougue e feira (onde, freqüência e quanto gasta), } \\
\text { quem faz a lista e efetua as compras }\end{array}$ \\
\hline Característica do cardápio & Definir junto com a dona da casa e com a cozinheira \\
\hline Avaliação antropométrica de todos os membros da família & Peso e estatura \\
\hline Proposta de mudança e conduta nutricional & Orientar quanto às mudanças emergenciais \\
\hline
\end{tabular}


da família. Este inquérito é aplicado com a dona da casa (ou responsável), que deverá responder a todas as informações relacionadas à alimentação da família. O objetivo do inquérito é conhecer bem todos os aspectos relacionados à saúde e aos hábitos de vida da família, tais como hábitos e comportamentos alimentares, peso e estatura dos indivíduos, exames bioquímicos, prática de exercício físico, práticas culinárias, rotina familiar, dentre outras informações relevantes.

Os dados coletados no inquérito familiar são importantes para definir a conduta nutricional e as metas a serem traçadas, e para elaborar o cardápio de acordo com os hábitos e a rotina familiar. As características do cardápio devem ser definidas em conjunto com a família. É importante ressaltar que as metas propostas e os cardápios prescritos sejam os mesmos para todos os membros da casa. Entretanto, pode ser necessária a individualização do consumo energético e da suplementação de nutrientes para algum membro da família. Nesse caso, a ferramenta mais importante é a anamnese individual (Quadro 2), que deve conter o máximo de informações possíveis sobre a situação de nutrição e saúde do indivíduo ${ }^{13}$.

\section{Primeira visita}

Para a primeira visita, é necessário que todos os moradores da casa estejam presentes, incluindo os funcionários que têm relação direta com a alimentação da família. Durante esta visita são identificados todos os aspectos relacionados à alimentação da família e, a partir do objetivo principal, elaboram-se as propostas de mudanças. Durante a primeira visita é o momento adequado para as seguintes abordagens: oferecer todos os serviços de personal dieter, propor um plano de acompanhamento de, no mínimo, dois meses, definir a periodicidade das visitas, realizar o inquérito familiar e a avaliação física dos membros da família, e orientar a cozinheira sobre as mudanças gerais na rotina culinária. O tempo médio gasto para esta visita é de duas horas e os materiais usados são: balança eletrônica portátil, adipômetro, fita métrica e material impresso (inquérito familiar, anamnese individual e bloco de prescrição).

\section{Propostas de mudanças}

As propostas de mudanças devem ser formuladas a partir de um criterioso diagnóstico

Quadro 2. Tipos de informações a serem coletadas pelo personal dieter mediante anamnese individual.

\begin{tabular}{|c|c|}
\hline Tipo de informação & Especificação \\
\hline Identificação do paciente & Nome, sexo, data de nascimento, endereço e objetivo principal \\
\hline Hábitos de vida e saúde & $\begin{array}{l}\text { Hábito de fumar, atividade física, sono, disposição ao longo do dia, } \\
\text { antecedentes do cliente e dos familiares em relação a doenças, cirur- } \\
\text { gias, medicação e suplementação }\end{array}$ \\
\hline Hábitos alimentares e fatores relacionados & $\begin{array}{l}\text { Preferências e aversões alimentares; ingestão de líquido ao longo do } \\
\text { dia; alergias conhecidas a medicamento, alimento ou inseto; freqüên- } \\
\text { cia a bares, delivery e lanchonetes }\end{array}$ \\
\hline Ciclo menstrual & $\begin{array}{l}\text { Regularidade, fluxo, data da última menstruação, tensão pré-mens- } \\
\text { trual, uso de anticoncepcional, amenorréia e menopausa }\end{array}$ \\
\hline Exames bioquímicos & $\begin{array}{l}\text { Hemograma completo, coprológico de fezes, dosagem sangüínea de } \\
\text { proteínas totais e frações, colesterol total e frações, triglicerídeos, creati- } \\
\text { nina, uréia, glicemia e hormônio estimulante da tireóide }\end{array}$ \\
\hline Dieta & Recordatório de $24 \mathrm{~h}$ e freqüência de ingestão de alimentos \\
\hline Sinais e sintomas gastrintestinais e correlacionados & $\begin{array}{l}\text { Hábito intestinal, flatulência, dores abdominais, digestão, aftas, dores } \\
\text { de cabeça, tontura, unhas quebradiças ou frágeis, manchas brancas e } \\
\text { estrias, inchaço, transpiração excessiva, mau hálito, bruxismo etc. }\end{array}$ \\
\hline Antropometría & Peso, estatura, pregas cutâneas e circunferências \\
\hline
\end{tabular}


da realidade, que, em geral, exige ações de prevenção e recuperação da saúde. Entretanto, podem haver casos de famílias que, aparentemente, não apresentam problemas alimentares e nutricionais, e que solicitam o serviço de consultoria para, por exemplo, planejar e variar cardápios e aperfeiçoar práticas culinárias. Porém, este tipo de clientela pode apresentar, de forma inconsciente, hábitos e comportamentos alimentares inadequados. Cabe ao nutricionista, por meio de um inquérito detalhado, identificar todos os

Primeira semana: concentrar na mastigação

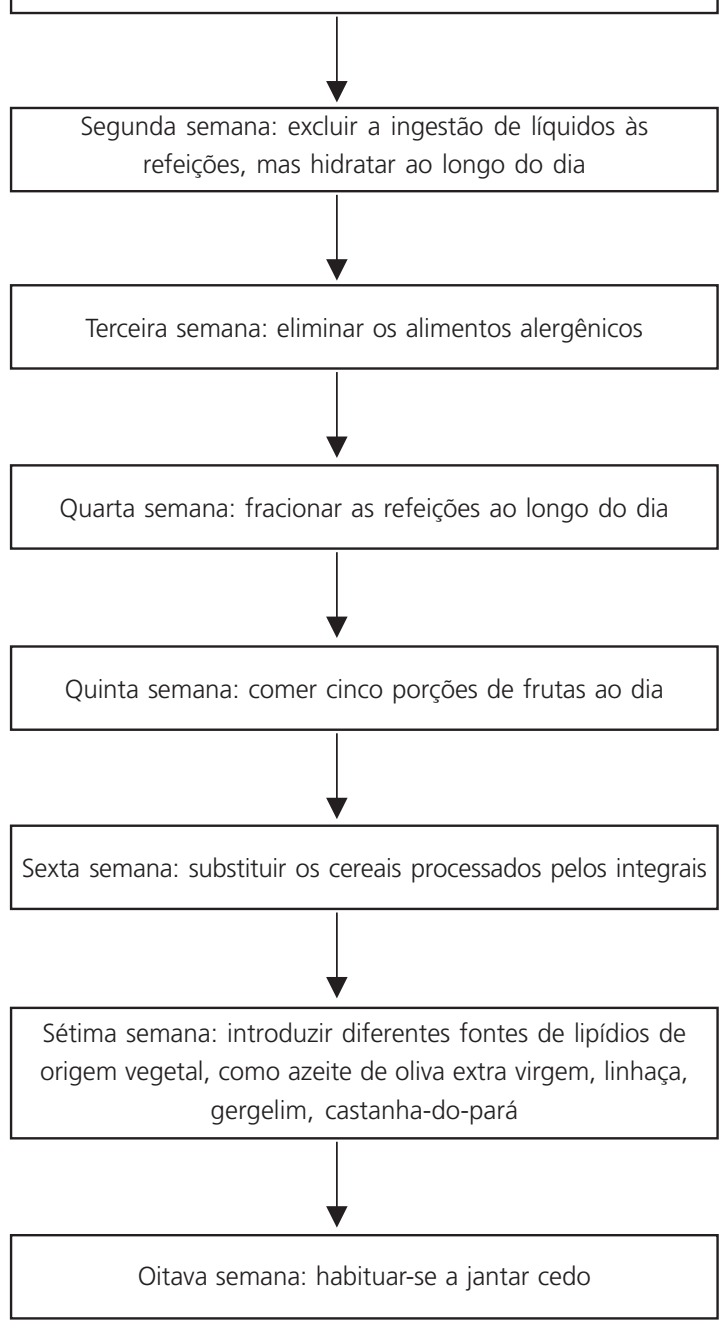

Figura 1. Fluxograma de uma proposta de intervenção para mudança de hábito alimentar por etapas, em consultoria nutricional familiar?. aspectos envolvidos com a alimentação e a nutrição da família, os quais determinarão os níveis de intervenção visando à saúde e à qualidade de vida dos clientes.

Na Figura 1 está apresentado um esquema de intervenção para mudança de hábitos alimentares incorretos, que freqüentemente são observados nas famílias. Todavia, é necessário o estabelecimento de estratégias diferenciadas e personalizadas de intervenção. Para isso, a competência e a criatividade do nutricionista são atributos essenciais. Por exemplo, caso a família não apresente problemas de mastigação e de ingestão de líquidos às refeições, mas consuma alimentos fora dos horários das refeições e em locais inapropriados, estes hábitos deverão ter prioridade no esquema de intervenção.

Para as famílias incorporarem os novos hábitos e atingirem as metas traçadas, é necessário tempo para adaptação às novidades, de modo que as mudanças devem ser propostas gradativamente. Os novos hábitos alimentares só serão incorporados se houver impacto positivo na saúde dos indivíduos e o objetivo principal for alcançado. Alguns aspectos são importantes para que ocorram as mudanças necessárias: discutir com a família os problemas identificados e as estratégias propostas para os enfrentar, repassar as informações de forma objetiva e clara, transmitir segurança e confiança durante a comunicação e propor mudanças de acordo com a realidade e por fases ou estágios ${ }^{11,13,16}$. É fundamental a priorização de metas e a implantação de correções e adaptações necessárias, em prazos bem definidos.

\section{Dieta individualizada}

Por meio de um software de Nutrição pode-se planejar a dieta do cliente baseada no cardápio familiar, mas com valor energético total específico. A dieta deve ser bem fracionada, composta de três refeições principais e três lanches intermediários. Pode haver a necessidade da inclusão de um ou dois lanches no decorrer do dia. O importante é que o indivíduo se alimente a cada três 
horas. Para facilitar o consumo dos alimentos em quantidades adequadas, recomenda-se que o prato do cliente que está submetido à dieta individual seja porcionado à francesa, pela cozinheira ou por outra pessoa da casa, uma vez que o tamanho das porções constitui um fator importante no controle do peso ${ }^{17}$. Além de estimar o valor energético da dieta e sua distribuição em macronutrientes, é recomendável considerar, no planejamento da dieta individual, os princípios da nutrição funcional, que visa recuperar o processo digestivo, promover a detoxificação hepática e o equilíbrio orgânico, e recuperar o estado nutricional do indivíduo, restabelecendo suas reservas orgânicas ${ }^{18,19}$. Assim, no início da intervenção pode ser necessária a suplementação de nutrientes e de outras substâncias para acelerar o processo de recuperação do trato gastrintestinal, como prebióticos, probióticos e enzimas digestivas ${ }^{18-20}$. Deve-se explicar ao cliente a finalidade e os mecanismos de ação de cada suplemento, de modo simplificado e claro.

\section{Segunda visita}

Após o período de uma semana, deverá ocorrer a segunda consulta. Nesta visita, verificam-se quais metas foram efetivamente alcançadas e se houve melhora na qualidade da alimentação, conforme a proposta estabelecida. Durante esta consulta deverá ser entregue o cardápio dos 15 primeiros dias e a respectiva lista de compra de alimentos, além da dieta individual, quando for o caso.

\section{Cardápio e lista de compra de alimentos}

Na entrega do cardápio, é efetuado o primeiro contato com a cozinheira. Nessa ocasião, devem ser tratados os seguintes temas: relação dos alimentos com a nutrição e a qualidade de vida; função de ingredientes, como açúcares simples, gorduras e temperos no preparo dos alimentos; e a importância da gastronomia no desenvolvimento de formulações, na aplicação de técnicas de cocção que permitam minimizar o uso de açúcares, gorduras e sal, e na manutenção da qualidade sensorial dos alimentos ${ }^{8}$.

A lista de compra de alimentos é elaborada a partir do cardápio. Essa lista constitui mais uma ferramenta de educação e de mudança dos hábitos alimentares. O cliente começa a perceber a qualidade da alimentação por meio dos produtos alimentares incluídos na lista, que deve contemplar a redução gradativa de produtos industrializados e o aumento de produtos in natura. Este planejamento de compras tem impacto não somente na saúde, mas também na economia doméstica, pois evita a aquisição de alimentos supérfluos e de alimentos que não serão usados no cardápio, e o decorrente desperdício, e também auxilia na previsão de custos. A lista de compra de alimentos pode ser dividida em hortifruti e em alimentos diversos. A pessoa responsável por essa compra deve ser orientada a ler os rótulos dos alimentos e a observar seus ingredientes, a fim de selecionar alimentos de melhor qualidade nutricional.

\section{Terceira visita}

A terceira visita é destinada à entrega do restante do cardápio para um mês e à realização de uma nova avaliação física. Nesta visita também podem ser oferecidos o treinamento para a cozinheira e a aula de educação nutricional.

\section{Treinamento da cozinheira}

O treinamento tem a finalidade de capacitar a cozinheira em técnicas dietéticas que garantam a produção de refeições com qualidade, e deve abordar vários aspectos, tais como: higiene dos alimentos; técnicas adequadas de armazenamento, refrigeração, congelamento, descongelamento, pré-preparo e cocção; reaproveitamento de alimentos; montagem de pratos; uso de ervas e condimentos e dicas de culinária. Os objetivos do treinamento são traçados conforme os proble- 
mas identificados no inquérito familiar ou durante as visitas domiciliares. Os aspectos que necessitam ser melhorados são, em geral, processos de higiene, quantidades de sal e de óleo usadas no preparo dos alimentos, desperdícios de alimentos e técnicas dietéticas básicas.

O treinamento da cozinheira pode ser dividido em três aulas, sendo duas teóricas e uma prática. É interessante fornecer uma apostila do conteúdo abordado nas aulas, elaborada de forma objetiva, clara e didática, por exemplo, contendo destaques e ilustrações para conteúdos relevantes. Ao final, deve ser aplicado um teste de conhecimentos e, caso o desempenho seja satisfatório, deve ser fornecido um certificado do treinamento.

\section{Aulas de educação nutricional}

O personal dieter precisa estar atento durante as visitas, que, muitas vezes, tendem a se tornar extensas, o que pode comprometer a eficácia do atendimento. As dúvidas e os questionamentos dos clientes são suficientes para demandar mais tempo do que o estipulado para uma consulta, sobretudo se houver necessidade de intervenções individuais. Por isso, devem ser sugeridas aulas de educação nutricional. As aulas constituem uma ferramenta importante para o profissional esclarecer melhor as dúvidas dos clientes, reforçar as orientações dadas durante as visitas domiciliares e abordar temas polêmicos sobre alimentação e nutrição, em geral veiculados de forma distorcida pela mídia7,8.

Recomendam-se as seguintes estratégias para o desenvolvimento das aulas: selecionar o conteúdo de acordo com o interesse, a idade e o objetivo principal da clientela, e com a proposta de intervenção; e expor o conteúdo de forma clara, dialogada e atrativa, usando recursos didáticos diversos como diapositivos, filmes educativos, folders, cartazes e pôsteres ${ }^{21}$. A duração de cada aula depende de vários fatores, como o número de pessoas e de perguntas, podendo variar de uma a duas horas.
No caso de haver muitas dúvidas e dificuldades em relação à mudança de hábitos alimentares, é conveniente propor à clientela uma oficina de culinária para auxiliar no processo de educação nutricional, estratégia que pode contribuir significativamente para a promoção da alimentação saudável13,22.

\section{CONSIDERAÇÕES FINAIS}

O serviço de consultoria alimentar e nutricional se torna diferenciado por vários aspectos. Em primeiro lugar, o paciente passa a ser cliente a partir do momento em que não precisa esperar para ser atendido, como acontece no atendimento em consultório. Ao contrário, o nutricionista personal dieter é que vai até a casa do cliente. A espera para o atendimento pode ser um fator que conduz à desistência de uma próxima consulta.

$\mathrm{O}$ acesso direto à rotina do cliente, o contato com o ambiente no qual os alimentos são armazenados, preparados e consumidos, a oportunidade de orientar quem manipula os alimentos, e o envolvimento da família no processo de mudança de hábitos alimentares constituem aspectos que diferenciam esse tipo de atendimento. Assim, o serviço do personal dieter possibilita uma maior adesão dos clientes à intervenção visando à recuperação do estado nutricional e, sobretudo, à promoção da saúde e da qualidade de vida da família.

\section{COLABOBORADORES}

P.A. ALMEIDA-BITTENCOURT e P.S.A. RIBEIRO contribuíram substancialmente com a coleta de dados e a redação do trabalho, que relata as estratégias de atuação das autoras em seis anos de experiência como nutricionistas na área de consultoria nutricional da família. M.M.V. NAVES orientou a concepção do trabalho (idéia e planejamento) e contribuiu com a redação e a revisão crítica do manuscrito

\section{REFER Ê NCIAS}

1. Brasil. Conselho Federal de Nutricionistas. Inserção profissional dos nutricionistas no Brasil. Brasília: CFN; 2006. 
2. Vasconcelos FAG. O nutricionista no Brasil: análise histórica. Rev Nutr. 2002; 15(2):127-38. doi: 10.1590/S1415-52732002000200001.

3. Monteiro CA, Mondini L, Costa RBL. Mudanças na composição e adequação nutricional da dieta familiar nas áreas metropolitanas do Brasil (1988-1996). Rev Saúde Pública. 2000; 34(3): 251-8. doi: 10.1590/S0034-89102000000300007.

4. Bleil SI. O padrão alimentar ocidental: considerações sobre a mudança de hábito no Brasil. Cad Debate. 1998; 6:1-25.

5. Lima A, Guerra NB, Lira BF. Evolução da legislação brasileira sobre rotulagem de alimentos e bebidas embalados, e sua função educativa para promoção da saúde. Hig Aliment. 2003; 17(110):12-7.

6. Rosser WW. Nutritional advice in Canadian family practice. Am J Clin Nutr. 2003; 77(4 Suppl):1011S-5S.

7. Viana V. Psicologia, saúde e nutrição: contributo para o estudo do comportamento alimentar. Anál Psicol. 2002; 4(20):611-24.

8. Bittencourt P, Ribeiro PA. Comer bem! Como? Manual de orientações nutricionais desde a compra até o preparo dos alimentos. Belo Horizonte: Leitura; 2008.

9. Ramalho RA, Saunders C. O papel da educação nutricional no combate às carências nutricionais. Rev Nutr. 2000; 13(1):11-6. doi: 10.1590/S1415-52 732000000100002.

10. McDonald HP, Gard AX, Haynes RB. Interventions to enhance patient adherence to medication prescriptions. J Am Med Assoc. 2002; 288(22): 2868-79. doi:10.1001/jama.288.22.2868.

11. Assis MAA, Nahas MV. Aspectos motivacionais em programas de mudança de comportamento alimentar. Rev Nutr. 1999; 12(1):33-41. doi: 10.15 90/S1415-52731999000100003.

12. Coyne T, Olson M, Bradham K, Garcon M, Gregory $P$, Scherch L. Dietary satisfaction correlated with adherence in the modification of diet in renal disease study. J Am Diet Assoc. 1995; 95(11):1301-6. doi:10.1016/S0002-8223(95)00341-X.

13. Gedrich K. Determinants of nutritional behavior: a multitude of levers for successful intervention?
Appetite. 2003; 41(3): 231-8. doi:10.1016/j.appet. 2003.08.005.

14. Williams MT, Hord NG. The role of dietary factors in cancer prevention: beyond fruits and vegetables. Nutr Clin Pract. 2005; 20(4):451-9. doi: 10.1177/01 15426505020004451.

15. Silva SMCS, Bernardes SM. Cardápio: guia prático para a elaboração. São Paulo: Atheneu; 2001.

16. Rodrigues EM, Soares FPTP, Boog MCF. Resgate do conceito de aconselhamento no contexto do atendimento nutricional. Rev Nutr. 2005; 18(1): 119-28. doi: 10.1590/S1415-527320050001000 11.

17. Jeffery RW, Rydell S, Dunn CL, Harnack $L$, Levine AS, Pentel PR, et al. Effects of portion size on chronic energy intake. Int J Behav Nutr Phys Act. [e-journal]. 2007 [cited 2007 June 27]; 4: 27 [5 p.]. Available from: http://www.ijbnpa.org/content/4/ 1/27. doi:10.1186/1479-58 68-4-27.

18. Biondo-Simões MLP, Mandelli KK, Pereira MAC, Faturi JL. Opções terapêuticas para doenças inflamatórias intestinais: revisão. Rev Bras Coloproctol. 2003; 23(3):172-82.

19. Paschoal V, Naves A, Fonseca ABBL. Nutrição clínica funcional: dos princípios à prática clínica. São Paulo: VP Editora; 2007.

20. Collins MD, Gibson GR. Probiotics, prebiotics and synbiotics: approaches for modulating the microbial ecology of the gut. Am J Clin Nutr. 1999; 69(5 Suppl):1052S-7S.

21. Holli BB, Calabrese RJ. Communication and education skills: the dietitian guide. Philadelphia: Lea \& Febiger; 1991.

22. Castro IRR, Souza TSN, Maldonado LA, Caniné ES, Rotenberg S, Gugelmin SA. A culinária na promoção da alimentação saudável: delineamento e experimentação de método educativo dirigido a adolescentes e a profissionais das redes de saúde e de educação. Rev Nutr. 2007; 20(6):571-88. doi: 10.1590/S1415-52732007000600001.

Recebido em: 13/12/2007

Versão final reapresentada em: 29/9/2008 Aprovado em: 10/6/2009 